\title{
MANIFESTATIONS OF TWO BRANCHES OF SOLAR ACTIVITY IN THE HELIOSPHERE AND GCR INTENSITY
}

\author{
M.B. Krainev \\ Lebedev Physical Institute, RAS, \\ Moscow, Russia,mkrainev46@mail.ru
}

\begin{abstract}
This paper provides insight into heliospheric processes and galactic cosmic ray (GCR) modulation occurring due to the presence of two branches of solar activity in this solar layer. According to the topology of solar magnetic fields, these branches are called toroidal (active regions, sunspots, flares, coronal mass ejections, etc.) and poloidal (high-latitude magnetic fields, polar coronal holes, zonal unipolar magnetic regions, etc.). The main cause of different manifestations of the two branches on the solar surface and in the heliosphere is the layer at the base of the heliosphere in which the main energetic factor is the magnetic field. The magnetic fields of the poloidal branch, which have
\end{abstract}

\section{INTRODUCTION}

When describing solar cycle in the photosphere and lower layers of the Sun, two topologically different systems of solar magnetic fields (SMFs) are identified as: toroidal $B_{\varphi}^{\mathrm{t}}$ and poloidal $B_{r, \vartheta}^{\mathrm{p}}$. These two systems are connected by the field of solar plasma motion, namely by its differential rotation, convection, etc. (see, e.g., [Vainstein et al., 1980; Charbonneau, 2010]). In the characteristics observed in the photosphere, active regions with sunspots and flares, mid- and low-latitude unipolar magnetic regions, etc. are assigned to the toroidal branch of solar magnetic fields. The poloidal branch includes high-latitude coronal holes, zonal unipolar magnetic regions, etc. (e.g., [Bruzek, Durrant, 1980; Mackay, Yeates, 2012]). Properties of these two SMF systems are substantially different. Magnetic fields of sunspots can generally be represented as bipolar structures with dimensions $L^{\mathrm{t}}<<r$, where $r$ is the photospheric radius, and with the maximum strength $B^{\mathrm{t}}$ of the order of several kilogauss. Systems of magnetic fields of coronal holes have large dimensions (for high-latitude ones $L^{\mathrm{p}} \geq r$ ), low mean strength $B^{\mathrm{p}} \approx 1 \div 10 \mathrm{G}$ and are unipolar. The two SMF systems develop in antiphase, i.e. when the number and total area of sunspots with a period of $\sim 11$ years reach a maximum, the area of polar coronal holes is minimum, and the strength of high-latitude magnetic fields reverses sign. And vice versa, when the coronal hole area reaches a maximum, the number and area of sunspots are close to zero, and the polarity of leading and following sunspots of bipolar structures, which is associated with the sign of $B_{\varphi}^{\mathrm{t}}$ of subphotospheric fields, reverses sign. It should be stressed that both the SMF systems are essential for understanding solar activity variability within the framework of the dynamo theory [Charbonneau, 2010]. By solar cycle, its minima and maxima, necessity and difficulty of its prediction, we, however, usually mean characteristics of a larger scale but a lower intensity, therefore gain an advantage in penetrating into the heliosphere. A connection is shown between the poloidal branch and heliospheric characteristics (solar wind velocity field, size of the heliosphere, form of the heliospheric current sheet, regular heliospheric magnetic field and its fluctuations) that, according to modern notions, determine GCR propagation in the heliosphere.

Keywords: galactic cosmic rays, heliosphere, GCR modulation, toroidal and poloidal branches of solar activity

the toroidal branch of solar activity (e.g., [Hathaway, 2015]). This is mainly due to the much more powerful effects of this branch (flares, coronal mass ejections) in the atmospheric layers adjacent to the Sun.

Galactic cosmic rays (GCRs) are observed in the outer layer of the solar atmosphere - the heliosphere, with the longest series of measurements of their intensity available for Earth's orbit. Since the very discovery of the long-term variation in GCR intensity (the socalled 11-year variation (see, for example, [Dorman, 1957])), it has been formulated as a change in GCR intensity in antiphase with solar cycle (that is, as usual, cycle of sunspot activity or toroidal branch of solar activity). It was natural because at that time (1950s) there was not any specific information available on the heliosphere and GCR modulation in it.

A lot has changed since then. Concepts of supersonic solar wind (SW) and large-scale structure of heliospheric magnetic fields (HMFs) have been formulated (references in [Parker et al., 1979; Owens, Forsyth, 2013]), long-term measurements of SW, HMF, and GCR characteristics near Earth and even in the highlatitude and interstellar transition heliospheric regions have been accumulated [Lazar, 2012; Balogh et al., 2014]. In particular, it has been shown that during most of the solar cycle the SW velocity varies greatly with heliolatitude, and the heliosphere is divided by the heliospheric current sheet (HCS) into two unipolar "hemispheres" according to HMF direction. In the GCR anisotropy and intensity, in addition to 11-year effects, 22-year effects have been found [Forbush, 1969; Ahluwalia, 1979]. On the other hand, the GCR modulation theory has predominantly been formulated which includes effects of GCR adiabatic deceleration and convection by the solar wind, their diffusion in irregular HMFs, as well as of particle drift in nonuniform HMF [Krymskiy, 1964; Parker, 1965; Jokipii et al., 1977]. The consideration of the 11-year cycle in sunspot activity as the main cause of the long-term GCR intensity variations in the 
heliosphere is still used and repeated from article to article (see, e.g., [Potgieter., 2013; Gerasimova et al., 2017]). In this case, the observed insignificant 22-year effects in GCRs are related to the variation with this period of preferred HMF polarity by magnetic drift ( $A$ is the sign of the radial HMF component in the northern hemisphere of the heliosphere) [Jokipii et al., 1977]. This viewpoint on the role of the two branches of solar activity in the heliosphere and GCR modulation in it has been analyzed in [Krainev et al., 2015].

As the author's research interests since the mid1970s have been in studying the effect of the discovered division of the heliosphere into two unipolar hemispheres on the heliosphere and GCR intensity [Shulz, 1973], the influence of magnetic drift of particles on GCR intensity [Jokipii et al., 1977], HMF inversion [Rosenberg, Coleman, 1969], etc., he naturally took the viewpoint on the role of the two branches of solar activity in the heliosphere, which was radically different from the above one.

This viewpoint developed in two ways. On the one hand, when the GCR modulation in the heliosphere was studied theoretically, the effects of the poloidal branch (particularly, of the magnetic drift) on the 11-year and 22-year GCR intensity variations were analyzed numerically. By excluding the drift term from the GCR modulation equation, the author suggested identifying the components associated with the two branches of solar activity in the GCR intensity. Each of these branches was shown to contribute significantly to both 11-year and 22-year intensity variations (see references in [Kalinin, Krainev, 2013, Krainev, Kalinin, 2013a]). To determine mechanisms of this process, a method of decomposing the estimated intensity into partial intensities, determined by different GCR modulation mechanisms, has been proposed [Krainev, Kalinin, 2013a; Krainev, 2015]. A hypothesis has been put forward that the particle drift affects other processes (diffusion, convection, adiabatic deceleration of GCR particles) by producing an intensity gradient [Krainev, 2013; Krainev et al., 2015]. It is important here that unlike diffusion that is determined by the presence of the intensity gradient when particles are transported toward the gradient, diminishing it, the drift causes the particle transport perpendicular to the gradient and can considerably change it without diminishing.

On the other hand, on the basis of the analysis of data on solar and heliospheric characteristics important for GCR propagation, we examined the formation of the heliosphere and its structure, the role of heliospheric boundary layers - adjacent to the Sun (in [Krainev, Webber, 2004], we called it the basement of the heliosphere) and adjacent to interstellar space (the so-called inner heliosheath). First, when discussing the effect of the two branches of solar activity on the heliospheric characteristics important for GCR, the consideration was largely qualitative [Krainev et al., 2013]. Then, using the model of potential magnetic field with source surface (see [Altschuler, Newkirk, 1969; Schatten et al., 1969] and references in [Hoeksema, 1984]), we determined the form of magnetic field neutral lines on the source surface, which after the line is extended by the solar wind in the heliosphere yields an acceptable model of the form of the heliospheric current sheet [Krainev, Kalinin, 2010]. Krainev, Kalinin [2013b] have examined characteristics of these forms, their variation with sunspot cycle phase, proposed their classification and HMF inversion model. Finally, in [Krainev, 2017; Krainev et al., 2019] the authors have briefly formulated arguments that virtually all SW and HMF characteristics important for GCR propagation during moderate and low sunspot activity periods are largely determined by the manifestation of the poloidal branch of solar activity in the heliosphere, which peaks in this period. Clearly, the GCR intensity defined by these heliospheric characteristics should also depend on the poloidal branch of solar activity in the above solar cycle phases.

As already noted, the viewpoint on the heliospheric processes as defined by both the branches of solar activity is shared by few. Especially important is, therefore, the appearance of the works [Bilenko, Tavastsherna, 2016; Bilenko, 2018] that consistently try to separate contributions of the toroidal and poloidal branches to the behavior of the heliospheric characteristics observed near Earth. This viewpoint is implicitly developed also in [Wang, 2014].

This paper discusses in more detail the ideas about the dependence of the solar and heliospheric characteristics important for GCR on the two branches of solar activity. General regularities of long-term variations in solar activity and GCR intensity are discussed in Section 1. Section 2 examines features of the heliosphere as an outer layer of the Sun; Section 3 identifies its characteristics important for GCR propagation. Section 4 deals with the dependence of characteristics of the heliosphere and CR intensity on both the branches of solar activity. This dependence is considered qualitatively, i.e. without formulating models of heliospheric characteristics, solving the GCR modulation equation, etc. Specific models of characteristics used in studying GCR propagation in the heliosphere, solution of the corresponding boundary value problem, and analysis of the solution to identify effects of each of the branches of solar activity are examined separately. Finally, Section 5 presents the results and conclusions obtained in the paper.

\section{LONG-TERM VARIATIONS OF SOLAR CHARACTERISTICS AND GCR INTENSITY}

To address the question raised in this paper as to what causes the long-term GCR intensity variations in the heliosphere, let us compare in Figure 1 the time dependences of solar activity and GCR intensity characteristics over the past 60 years.

We can see that the two branches of solar activity generally develop in antiphase: at sunspot activity maximum, poloidal magnetic fields are minimum and exhibit inversion. And vice versa, minimum sunspot areas and inversion of their associated toroidal magnetic fields (manifested as reversal of the polarity of leading and following sunspots in a new cycle) are observed during the periods when high-latitude SMFs are maximum. 


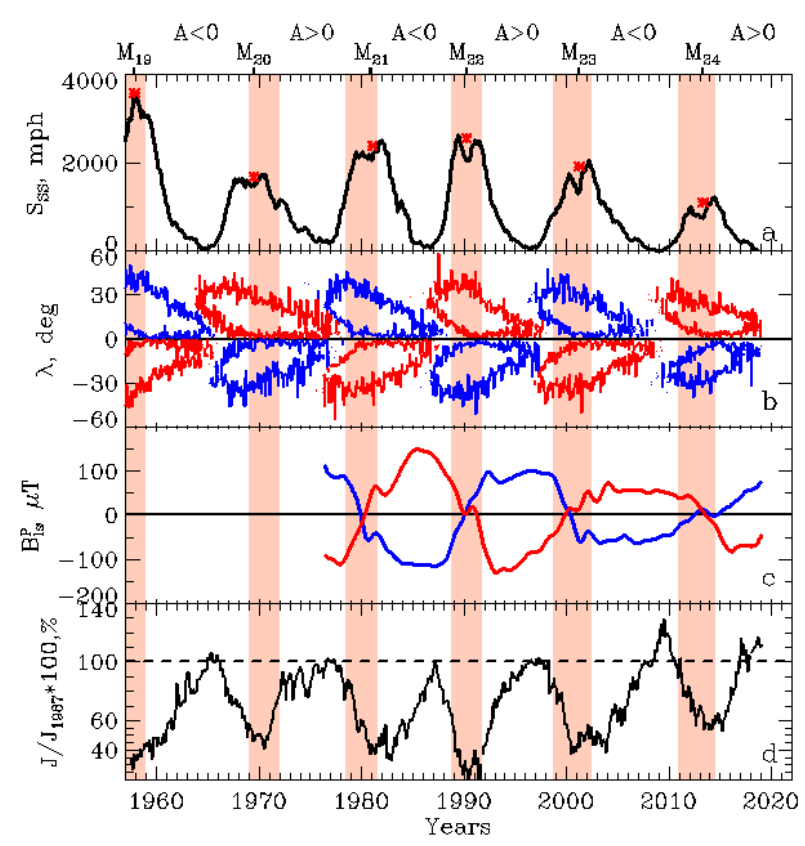

Figure 1. Time dependence of characteristics of two branches of solar activity and GCR intensity near Earth's orbit in 1957-2019. Periods of HMF inversion (see Subsection 4.2.) are indicated by vertical shaded stripes, and over the panels are moments of sunspot maxima $\left(M_{N}\right.$ for the $N$ th cycle according to the Zurich classification) and predominant HMF polarity $A$. All the characteristics except for those shown in panels $b$ and $d$ are 1-year smoothed. Panel $a$ shows the total sunspot area for two hemispheres [https://solarscience.msfc.nasa.gov], asterisks mark the sunspot area average for two Gnevyshev peaks; boundaries of latitudinal zones of bipolar active areas with $B_{\varphi}>0$ (blue lines) and $B_{\varphi}<0$ (red lines) [https://solarscience. msfc.nasa.gov] (b); the high-latitude photospheric magnetic field component along the line of sight in the northern and southern solar hemispheres (black and lighter lines respectively) [http://wso.stanford.edu] (c); the medium-energy GCR intensity normalized by $100 \%$ in 1987 (protons with kinetic energy $T>100 \mathrm{MeV}$ ) as derived from data taken at [https://sites.lebedev.ru/en/DNS_FIAN] (see [Stozhkov et al., 2007]) (d)

Since there are normally two maxima (so-called Gnevyshev peaks with the Gnevyshev Gap between them [Gnevyshev, 1967; Bazilevskaya et al., 2000]) in the time profile of the total sunspot area, as an index of maximum sunspot area $S_{\mathrm{SS}}^{\max }$ we take the mean sunspot areas in these local maxima, indicated by asterisks for solar cycles 19-24 in Figure 1, $a$.

The comparison between panels $a$ and $d$ also shows that the 11-year variation in GCR intensity is generally in antiphase with sunspot activity. Thus, it is clear on what the viewpoint that the 11-year GCR intensity variation (its amplitude and phase) is largely determined by sunspot activity is based. At the same time, it is evident that different HMF polarities during successive periods of low sunspot activity show up in the time profile of GCR intensity: the time profile of GCR proton intensity is more acute at $A<0$ and more plateau-like at $A>0$.

These details are usually assigned to the effects of the total solar magnetic field as if it exists in addition to solar cycle.
Figure 1 suggests that the HMF polarity reversal to which GCRs respond occurs during maximum sunspot activity periods close to periods of inversion of the poloidal branch of solar activity (Figure 1,c) rather than of the toroidal one (Figure 1,b). This means that for some reason features of the poloidal branch, which is less pronounced on the Sun than the toroidal one, manifest themselves in the heliosphere. This fact seems to us to be the main evidence for the active role of the poloidal branch of solar activity in processes occurring in the heliosphere, including GCR modulation.

\section{FEATURES OF THE HELIOSPHERE AS AN OUTER LAYER OF THE SUN}

To understand features of the heliosphere, it is worth considering the heliocentric distance dependence of SW and HMF energy density, shown in Figure 2, $a$. Note that the above dependence is valid only to some extent - in fact both the Sun and the heliosphere are spherically asymmetric, and distribution of the characteristics along the ray emanating from the Sun will depend on the direction of this ray, as well as on the solar cycle phase, time within this phase, etc.

Referring to Figure 2, $a$, the photosphere exhibits an approximate equality of densities of thermal $(\varepsilon T=n k T$, where $n, T, k$ are the SW density and temperature and the Boltzmann constant) and magnetic energy $\left(\varepsilon_{\mathrm{B}}=\frac{B^{2}}{8 \pi}\right.$, where $B$ is the magnetic induction), which with distance away from the Sun due to decreasing SW density is quickly violated so that up to the distances of the order of $r$ $\mathrm{HS} \approx 10 r$ the main dynamic factor is the magnetic field. At the same time, in this layer, which we call, for brevity sake, the basement of the heliosphere, SW accelerates. The heliosphere extends from $r=r_{\mathrm{HS}}$ to $r=r_{\mathrm{HP}}$, where the main dynamic factor is the supersonic and super-Alfvén SW ( $\varepsilon_{\mathrm{SW}}=n m V^{2} / 2$, where $V$ is the $\mathrm{SW}$ velocity, $m$ is the proton mass). Next is the interstellar medium located very close to the Sun (Very Local Interstellar Medium, VLISM).

This spherically symmetric geometry of the heliosphere is violated by the solar motion with respect to the interstellar medium as well as by the heliolatitude dependence of SW characteristics, thus producing a complicated structure of the heliosphere containing the TS surface after which SW becomes subsonic in the heliosheath - the layer between TS and heliopause. If the motion of the solar system relative to the interstellar medium occurs at a supersonic speed, BS is formed in the interstellar medium. Figure 2, $b$, drawn from the calculations made in [Baranov, Malama, 1993], provides an insight into the real structure of the heliosphere.

Thus, for answering the question of interest about the relationship between manifestations of the two branches on the Sun and in the heliosphere, processes occurring in the basement of the heliosphere are of paramount importance. 

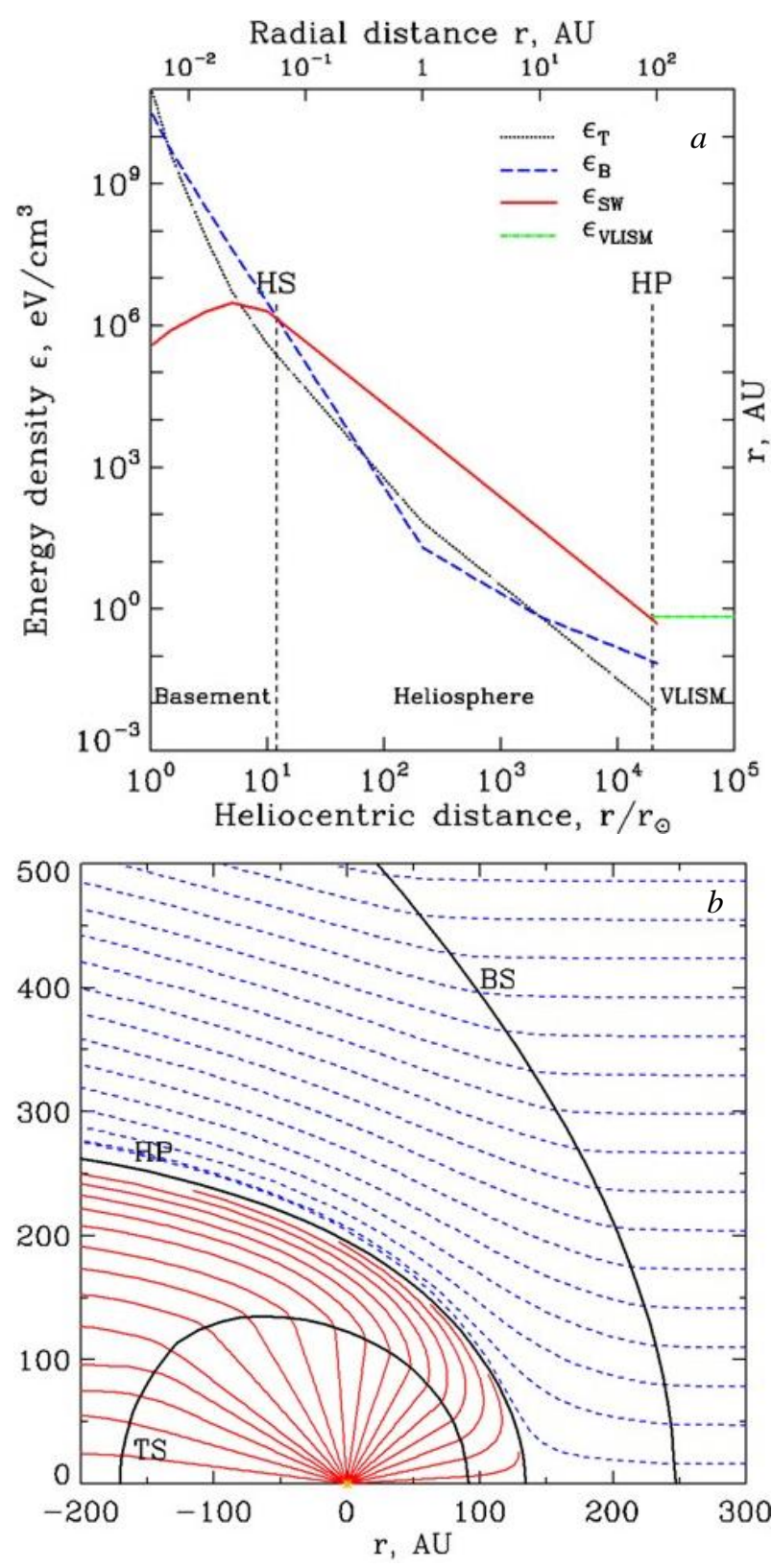

Figure 2. Features and structure of the heliosphere: density of energy of different types (thermal, magnetic, SW kinetic, interstellar) as a function of relative heliocentric distance $\left(r / r_{\odot}\right.$, where $r$ is the photosphere radius in the solar atmosphere as derived from data obtained mainly in [Rossi, Olbert, 1970] (a). Vertical dashed lines indicate inner (HS) and outer (HP) boundaries of the heliosphere; current lines of solar and interstellar winds (thin solid and dashed lines respectively) and the main surfaces of the heliosphere structure: terminal shock (TS), heliopause (HP), and bow shock of the interstellar wind (BS) as derived from the calculations made in [Baranov, Malama, 1993] (b)

\section{HELIOSPHERIC CHARACTERISTICS IMPORTANT FOR GCR PROPAGATION}

Before examining the effect of the two branches of solar activity on heliospheric characteristics, let us identify the most important of them for GCR propagation in the heliosphere. According to current view (see, e.g., the review [Potgieter, 2013] and references there- in), in a stationary case the GCR intensity $J(r, T)$ with kinetic energy $T$ and momentum $p$ is described by the solution of the boundary value problem with the following transport equation for GCR distribution function $U(r, p)=J(r, T) / p^{2}$ :

$$
\begin{aligned}
& -\nabla(\mathbf{K} \nabla U)+\mathbf{V}_{\mathrm{d}} \nabla U+\mathbf{V} \nabla U-(\nabla \mathbf{V} / 3) \times \\
& \times(\partial U / \partial \ln p)=0,
\end{aligned}
$$

boundary conditions on the inner $\left(r=r_{\min }\right)$ and outer $(r=$ $\left.r_{\max }\right)$ boundaries of the modulation region:

$$
\begin{aligned}
& \partial U /\left.\partial r\right|_{r=r_{\min }}=0, \\
& U_{r=r_{\max }}=U_{\mathrm{nm}}(p),
\end{aligned}
$$

where $U_{\mathrm{nm}}(p)$ is the unmodulated GCR distribution function in the interstellar medium very close to the Sun; as well as by the so-called initial condition - the absence of modulation for particles of sufficiently high energy $\left(T_{\max }=100 \mathrm{GeV}\right)$ :

$$
\left.U\right|_{p=p_{\max }}=U_{\mathrm{nm}}\left(p_{\max }\right) .
$$

In (1), $\mathbf{K}, \mathbf{V}, \mathbf{V}_{\mathrm{d}}$ are the particle diffusion tensor, $\mathrm{SW}$ velocity, and magnetic particle drift velocity respectively. The diffusion tensor is determined by induction of regular HMF $\mathbf{B}$ and by spectrum of its irregularities in wavenumbers $P(k)$. The magnetic drift velocity satisfies the expression

$$
\mathbf{V}_{\mathrm{d}}=\frac{p v}{3 q}\left[\nabla \times \frac{\mathbf{B}}{B^{2}}\right]
$$

where $v$ and $q$ are the particle velocity and charge.

Thus, in accordance with the purposes of this paper, it is necessary to determine the effect of these two branches of solar activity on variations of SW velocity, regular HMF strength and polarity, spectrum of its irregularities, as well as probably on the form of the GCR modulation region boundary.

\section{HELIOSPHERIC CHARACTERISTICS AND TWO BRANCHES OF SOLAR ACTIVITY}

While the most powerful heliospheric factor is the solar wind, we first examine processes occurring in the basement of the heliosphere and HCS form, rather than $\mathrm{SW}$ velocity. This choice is due to the fact that through the processes in the basement during most solar cycle both the SW velocity and the regular HMF distribution appear to be organized with respect to the distance from HCS.

\subsection{The simplest model of the magnetic field} in the basement of the heliosphere and HCS form

A model of the potential solar magnetic field with source surface is widely used to study GCR variations. The model is developed at the Wilcox Solar Observatory (WSO) [Schatten et al., 1969; Altschuler, Newkirk, 
1969; Hoeksema, 1984]) and suggests the complete absence of currents in the basement of the heliosphere, $r_{\odot} \leq r \leq r_{\mathrm{SS}}$, where $r_{\mathrm{SS}}$ is the HMF source surface radius - the outer boundary of the basement and the inner boundary of the heliosphere. Then, in the potential approximation for the scalar potential $\Phi=\sum_{l=0}^{l=9} \Phi_{l}$, satisfying the equation $\nabla^{2} \Phi=0$, the solar magnetic field induction $\mathbf{B}=\nabla \Phi$ in the basement of the heliosphere is represented as a series expansion in spherical functions

$$
\begin{aligned}
& \Phi_{l}=r \sum_{m=-l}^{m=l} P_{l}^{m}(\vartheta) \times \\
& \times\left\{g_{l m} \cos (m \varphi) C_{l m}(r)+h_{l m} \sin (m \varphi) D_{l m}(r)\right\}
\end{aligned}
$$

(слева в (6) должно быть $\Phi_{1}$ )

with known functions $P_{l}^{m}(\vartheta), C_{l m}(r), D_{l m}(r)$ and coefficients $g_{l m}, h_{l m}$, determined from results of daily scanning of the photospheric magnetic field component along the line of sight and boundary conditions at $r_{\odot}$ and $r_{\mathrm{SS}}$. On the website [http://wso.stanford.edu], two sets of these coefficients for two versions of the boundary condition at $r_{\odot}$ defining results of the WSO model are published for each Carrington rotation starting with CR 1642 (May-June 1976): classical when the whole component of SMF induction along the line of sight is used as the inner boundary condition, and radial when the SMF direction on the photosphere is assumed to be radial.

Equation (6) allows us to calculate all the three components of SMF induction at any point of the basement. It is necessary to mention certain drawbacks of the WSO model: 1) complete absence of currents in the basement, including current sheets; 2 ) source surface is located close to the Sun $\left(r_{\mathrm{SS}}=2.5 r_{\odot}, 3.25 r_{\odot}\right.$ for classical and radial versions of the model respectively), although SW becomes supersonic and super-Alfvén at much greater distances $\left(r_{\mathrm{HS}}>10 r_{\odot}\right)$. These drawbacks lead to the fact that 1) the radial component $B_{r}$ on the source surface appears to be several times weaker than it follows from its measurements at Earth's orbit and from the radial dependence inversely proportional to the squared heliocentric distance in the heliosphere; 2) an increase in the $B_{r}(\theta, \varphi)$ modulus with distance away from the current layer does not correspond to the constancy of $B_{r}(\theta, \varphi)$ outside HCS derived from the Ulysses data [Smith et al., 1995]. It has been shown [Schatten, 1971; Zhao, Hoeksema, 1994] that by complicating the models these drawbacks can be overcome, but the authors of the WSO model did not follow this way, and on the website [http://wso.stanford.edu] results of the simple model are still posted and widely used.

The absolute value and the dependence of the $B_{r}(\theta$, $\varphi)$ on coordinates in the source surface, calculated from the WSO model, are therefore not employed in the study of the GCR modulation in the heliosphere, but the form of the calculated neutral lines $B_{r}(\theta, \varphi)=0$ on this surface in the first approximation corresponds to the HCS form, as derived from Ulysses, Pioneers, Voyagers data [Balogh, Jokipii, 2009; Balogh, Erdos, 2013], and is widely used.
In the following sections, we make extensive use of the WSO model results of two types: form of neutral isolines $B_{r}(\theta, \varphi)=0$ on the source surface and the magnetic flux through the photosphere and source surface. In this case, when calculating $\mathbf{B}$ in the basement, we omit the monopole term $l=0$ in (6), although there are respective coefficients in the published sets. In addition, when calculating SMF fluxes we are interested in the contribution of the magnetic fields corresponding to different indices $l$ to these fluxes, particularly in the contribution of the dipole term $l=1$. The neutral isolines are considered as bases of heliospheric current sheets, and by the above fluxes we can judge on the processes occurring in the basement of the heliosphere.

We demonstrate the capabilities of the WSO model to describe SMF in the basement of the heliosphere and what occurs at the transition from the photosphere to the heliosphere, using one Carrington rotation (CR 2063, November 2007, the period of low sunspot activity) as an example. Consider first the distribution of different solar activity effects in the photosphere (coronal holes, filaments, plages, sunspots) compiled from McIntosh maps [https://www.ngdc.noaa.gov/stp/spaceweather/solar-data/solar-imagery/composites/synopticmaps/mc-intosh/ptmc_level3/ptmc_level3_gifs]. Figure 3 shows polar and low-latitude coronal holes and unipolar regions separated by filaments - the remains of SMFs of active regions. In the top panel of Figure 4 is the WSO-model-calculated SMF distribution over the photosphere for CR 2063. The WSO model is seen to reproduce the main features of the distribution of different solar activity effects by the SMF polarity, although the model distribution is much more blurred.

Consider now how the model distributions of $B_{r}$ change during the transition from the photosphere to the source surface, which are shown in the top and bottom panels of Figure 4 respectively. We can see that with increasing distance from the photosphere the distribution of magnetic fields over the basement is considerably simplified. Instead of alternating many unipolar SMF areas in the photosphere such as magnetic fields of active regions, coronal holes, etc., on the source surface there is only one curved neutral line during the period of interest, which splits the surface into two unipolar hemispheres, with the $B_{r}$ polarity in them corresponding to the polarity of high-latitude fields in the photosphere. At the same time, it is evident that the form of the neutral line is significantly affected by mid- and low-latitude unipolar regions, mainly by coronal holes.

This simplified distribution of SMF by polarity illustrates the main function of the basement - the filtration of magnetic fields by their characteristic dimensions during the transition from the photosphere to the heliosphere, giving advantages to large-scale magnetic fields even if they are much weaker. For us, this means that magnetic fields of the poloidal branch get an advantage in penetrating into the heliosphere as compared to those of the toroidal one. 


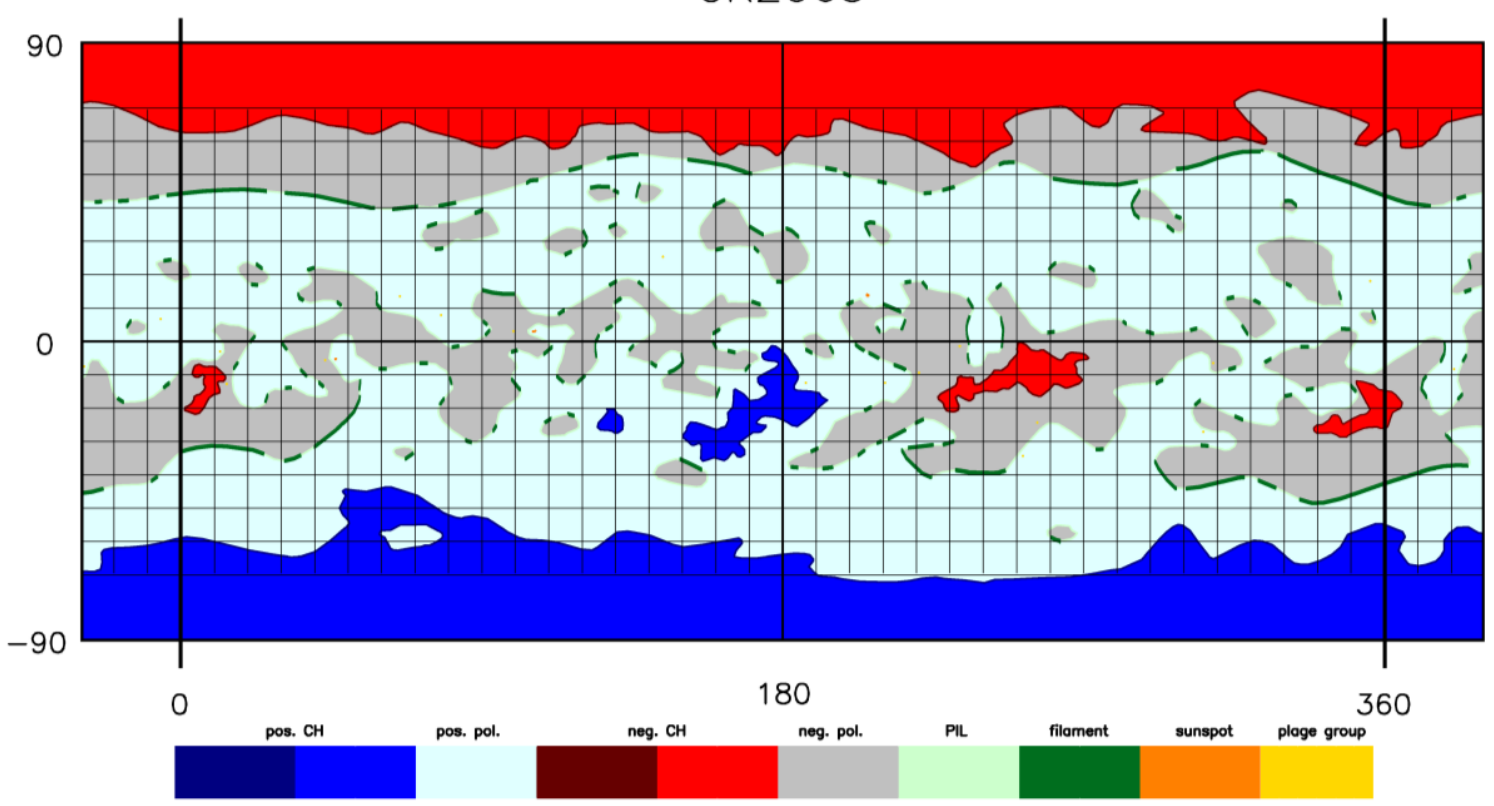

Figure 3. Distribution of different solar activity effects near the solar photosphere (McIntosh map) for CR 2063 [https:// www.ngdc.noaa.gov/stp/space-weather/solar-data/solar-imagery/composites/synoptic-maps/mc-intosh/ptmc_level3/ptmc_level3_gifs]. The fill color at the bottom corresponds to solar formations and magnetic field polarity

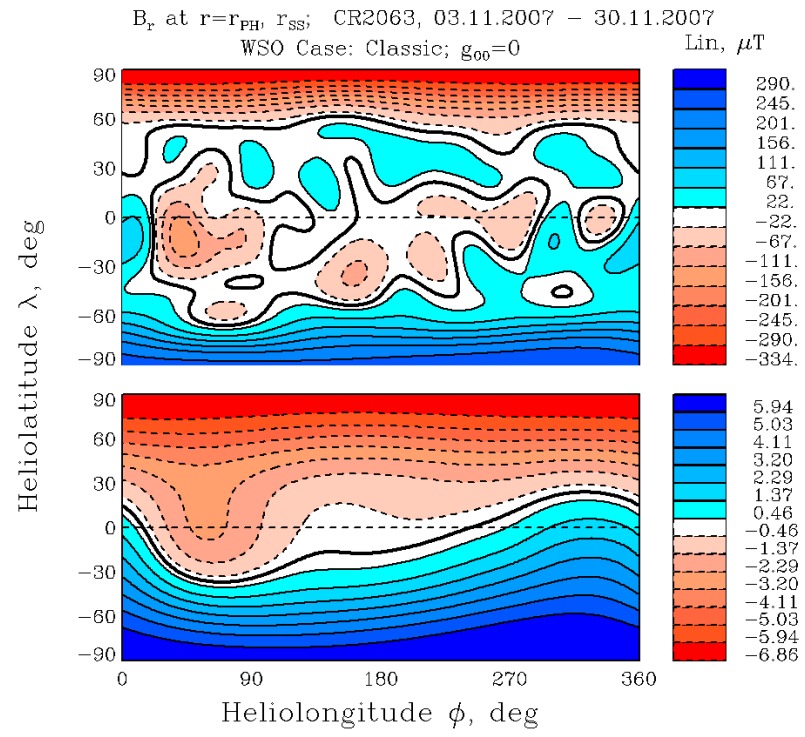

Figure 4. Distributions of the radial component of SMF induction over the solar photosphere (top panel) and over the HMF source surface (bottom panel) calculated by the WSO model [Hoeksema, 1984] for CR 2063 as in Figure 3. Isolines of $B_{r}=$ const are indicated by solid thin lines for $B_{r}>$ and by dashed lines for $B_{r}<0$; isolines of $B_{r}=0$, by thick solid lines. Narrow right panels show values of $B_{r}$ in different isolines

\subsection{Heliospheric current sheet form and HMF inversion}

Since during most solar cycle HCS organizes around itself polarity distributions of large-scale HMF and SW velocity and, hence, some other characteristics important for GCR, let us first consider the simple classification of HMF polarity distribution [Krainev, Kalinin, 2013b] and its change with solar cycle phase. Krainev, Kalinin [2013b] think that the following changes in the HMF polarity distribution comprise the HMF inversion the time limits of different phases of which for the last four solar cycles are summarized in Table (in Carrington rotations and approximately in months and years). In addition, the HMF polarity distribution in boundary rotations of the last HMF inversion in cycle 24 is shown in Figure 5. To draw Table and Figure 5, we have used the classical version of the WSO model. When another (radial) version of the WSO model is employed, there may be minor changes in the boundaries of HMF inversion phases.

Within about 7-8 of 11 years, the heliosphere was divided by the only global (i.e. covering all longitudes) HCS into two unipolar hemispheres. Examples of such HMF polarity distributions are given in panels $a$ and $e$ of Figure 5. Such an HMF distribution may be called dipole and described by the predominant polarity $A$ by the sign of $B_{r}$ in the northern hemisphere.

The dipole HMF phase represents epochs of moderate and low sunspot activity.

As the epoch of the maximum toroidal branch of solar activity approaches, in addition to the global HCS there appear local HCSs bounding the areas of opposite polarity within the unipolar hemispheres (Figure 5, b). Let us call the period of this HMF distribution the phase of their pre-inversion. Then, the global HCS is broken, after some time (HMF inversion phase, panels $c$ and $d$ ) it is 
formed again, but HMF polarity in each hemisphere is reversed. In this case, during several rotations (HMF postinversion phase, panel $e$ ) areas of opposite polarity may still exist in the unipolar hemispheres; then during the next 7-8 years there is the only global HCS (new HMF dipole phase, Figure 5, $f$ ).

Intervals outside the inversion periods listed in Table refer to continuous dipole phases of HMF with corresponding polarity lasting for $7-8$ years. The only exception, omitted in Table, is the emergence of a small area of negative polarity in the large positive hemisphere in Carrington rotation 2037 (November 24, 2005 - December 21, 2005).

During the HMF dipole phase, the degree of waviness of the global HCS changes. This degree is usually characterized by the so-called quasi-tilt value equal to half of the heliolatitude range occupied by the global HCS,

$$
\alpha_{\mathrm{qt}}=\frac{\lambda_{\max }^{\mathrm{HCS}}-\lambda_{\min }^{\mathrm{HCS}}}{2},
$$

where $\lambda_{\max }^{\mathrm{HCS}}$ and $\lambda_{\min }^{\mathrm{HCS}}$ are the maximum and minimum heliolatitudes of the global HCS. The $\alpha_{\mathrm{qt}}$ value is regularly published and widely used for analyzing the behavior of GCRs. Note that on the website [http://wso.stanford.edu] this characteristic is presented for both the versions of the WSO model and not only for the period of the dipole HMF. If there are several HCSs (panels $b, e$ in Figure 5), the maximum and minimum heliolatitudes are calculated from all existing HCSs. In our opinion, the use of the quasi-tilt for HMF inversion periods in the analysis and modeling of GCRs is difficult to justify.

Thus, the HMF inversion in phase with the inversion of poloidal, rather than toroidal, SMFs is important evidence of the influence of the poloidal branch of solar activity in the heliosphere. During the HMF inversion period close to the phase of the toroidal branch maximum, the effect of weak poloidal fields on SW and HMF strength is however small as compared to the maximum effect of the toroidal branch and its associated non-stationary phenomena (solar flares, coronal mass ejections, etc.). We therefore restrict ourselves to the HMF dipole phase when discussing the effect of the poloidal branch of solar activity on the behavior of characteristics important for GCRs.

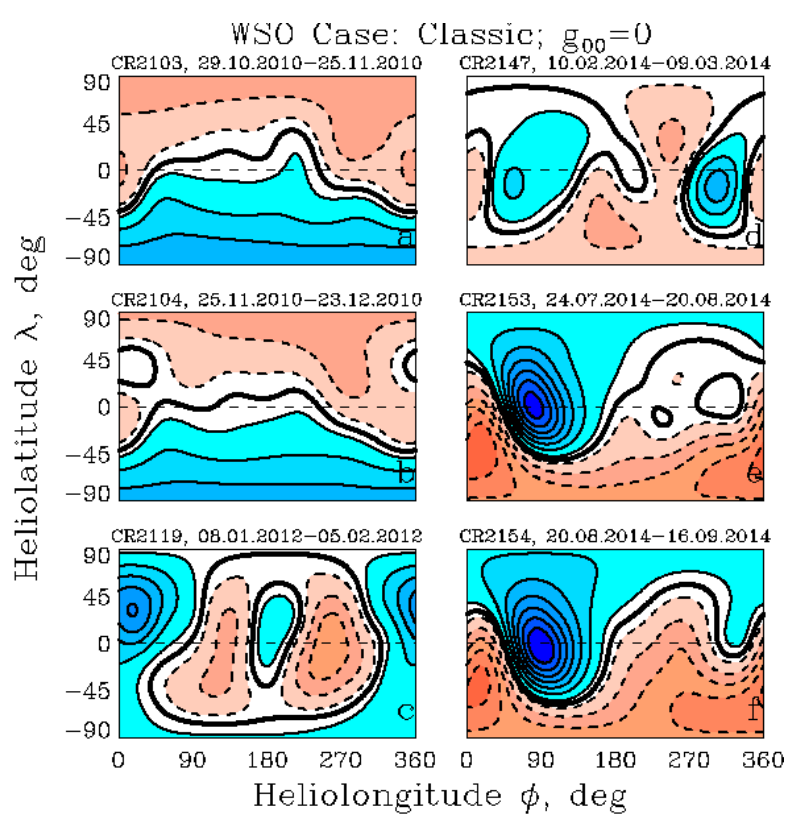

Figure 5. Change in the HMF polarity distribution during inversion through the example of cycle 24 . The last rotation of the HMF dipole phase before inversion $(a)$; the first rotation of the pre-inversion phase $(b)$; the first and last rotations of the HMF inversion phase $(c, d)$; the last rotation of the postinversion phase $(e)$; the first rotation of the new dipole HMF phase $(f)$

\subsection{Solar wind velocity field}

The organizing effect of HCS on the SW velocity field and HMF, discussed in the previous section, during much of the solar cycle in the first approximation means that at the base of the heliosphere 1) the SW velocity is minimum on HCS and increases rapidly with distance away from it [Hundhausen, 1972; Shulz, 1973], see also [Wang, Sheeley, 1990; Arge, Pizzo, 2000]; 2) the radial HMF component within the hemisphere is unipolar and at a certain heliocentric distance is uniform in each of the two hemispheres separated by the global HCS [Smith et al., 1995].

The above arrangement of SW fields and HMFs relative to the global HCS is well illustrated in Figure 6 based on Ulysses data (the third fast flight of Ulysses from the southern polar latitude to the northern one). We can see that the SW velocity becomes high; and HMF, unipolar and uniform in the radial component approximately at instants (indicated by vertical dashed lines) when the spacecraft goes out of the latitudinal zone occupied by the

Time limits of HMF inversion phases in the last four solar cycles

\begin{tabular}{|c|c|c|c|c|c|}
\hline $\begin{array}{c}\text { HMF } \\
\text { inversion } \\
\text { phase }\end{array}$ & $\begin{array}{c}\text { Solar cycle } \\
\text { HMF polarity } A \\
\text { before/after inver- } \\
\text { sion }\end{array}$ & $\begin{array}{l}\mathrm{SC} 21 \\
+/-\end{array}$ & $\begin{array}{c}\mathrm{SC} 22 \\
-/+\end{array}$ & $\begin{array}{l}\mathrm{SC} 23 \\
+/-\end{array}$ & $\begin{array}{c}\mathrm{SC} 24 \\
-/+\end{array}$ \\
\hline $\begin{array}{c}\text { pre-inversion } \\
\text { HMF }\end{array}$ & $\begin{array}{l}\text { Carrington rotations } \\
\text { month, year }\end{array}$ & $\begin{array}{c}1670-1682 \\
07.1978-06.1979\end{array}$ & $\begin{array}{c}1809-1813 \\
11.1988-03.1989\end{array}$ & $\begin{array}{c}1942-1952 \\
11.1998-08.1999\end{array}$ & $\begin{array}{c}2104-2118 \\
12.2010-12.2011\end{array}$ \\
\hline $\begin{array}{l}\mathrm{HMF} \\
\text { inversion }\end{array}$ & $\begin{array}{l}\text { Carrington rotations } \\
\text { month, year }\end{array}$ & $\begin{array}{c}1683-1693 \\
07.1979-03.1980\end{array}$ & $\begin{array}{c}1814-1834 \\
04.1989-10.1990\end{array}$ & $\begin{array}{c}1953-1958 \\
09.1999-01.2000\end{array}$ & $\begin{array}{c}2119-2147 \\
01.2012-02.2014\end{array}$ \\
\hline $\begin{array}{l}\text { HMF post- } \\
\text { inversion }\end{array}$ & $\begin{array}{l}\text { Carrington rotations } \\
\text { month, year }\end{array}$ & $\begin{array}{c}1694-1711 \\
04.1980-08.1981\end{array}$ & $\begin{array}{c}1835-1847 \\
11.1990-10.1991\end{array}$ & $\begin{array}{c}1959-1990 \\
02.2000-06.2002\end{array}$ & $\begin{array}{c}2148-2153 \\
03.2014-08.2014\end{array}$ \\
\hline
\end{tabular}


global HCS, calculated using the classical, not radial, version of the WSO model [URL WSO].

Thus, due to the influence of large-scale (mostly poloidal) SMFs in the basement of the heliosphere the SW velocity and density in huge heliospheric regions change approximately twice in phase with activity of the poloidal branch. Because of the change in the SW velocity and density, the size of the GCR modulation region may also change greatly, $r_{\max } \sim\left(n V^{2}\right)^{1 / 2}$.

Furthermore, these features lead both to a significant latitude dependence of the longitude-averaged SW velocity and to the formation of SW streams of different velocities in close longitudes in the latitudinal zone occupied by the global HCS. The streams can be clearly seen in the middle panel of Figure 6. As a consequence, this gives rise to regions of their interaction, enhanced HMF disturbance, etc. [Richardson, 2018].

\subsection{Magnetic fluxes and radial component of regular HMF}

Krainev [2017] has observed high amplitude of SW velocity and HMF variations generated by the poloidal branch of solar activity. The modulus of the HMF strength vector change during the transition from one maximum of the toroidal cycle to the next one (when HMF reverses forming the 22-year cycle) is compared with the variation in the HMF strength modulus in the sunspot (or 11-year) cycle. Thus, virtually throughout the heliosphere the HMF variation in the poloidal cycle is not smaller than that in the toroidal cycle.

As one of the main features of HMF distribution the previous section considers the constancy of the absolute value of $B_{r}(\theta, \varphi)$ at a certain heliocentric distance in each of the unipolar hemispheres, clearly shown in the bottom panel of Figure 6. Now figure out which of the two branches of solar activity is responsible for this very value.

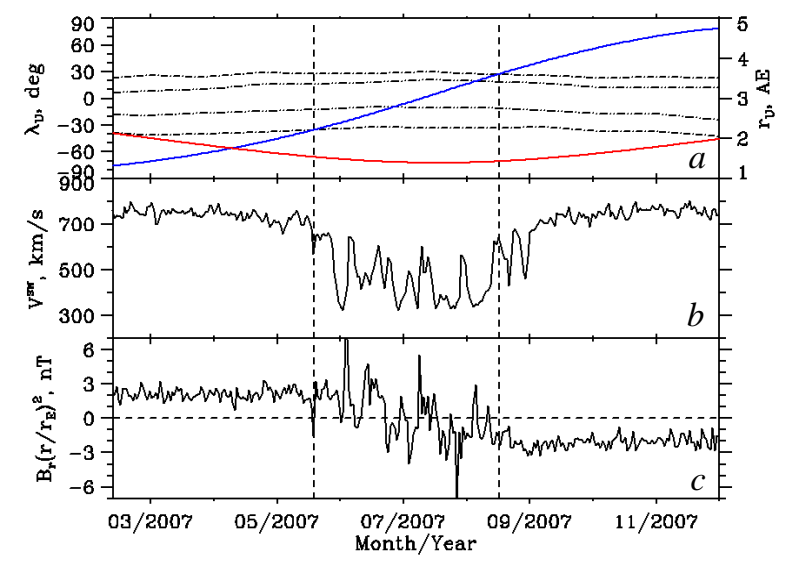

Figure 6. SW velocity (panel $b$ ) versus HMF radial component, reduced to $1 \mathrm{AU}$ (panel $c$ ), along the Ulysses trajectory [http://ufa.esac.esa.int/ufa/\#data]. Panel $a$ shows the behavior of the heliolatitude (solid line) and heliocentric distance (dashed line) of Ulysses, as well as maximum and minimum heliolatitudes of the global HCS for the classical (dash-dotted line) and radial (dash-dot-dot line) versions of the WSO model [http://wso.stanford.edu]
Figure 7 compares time variations in the solar magnetic flux through the photosphere, the source surface (provided that $B_{r}(\theta, \varphi)$ is constant) and the sphere $r=1$ $\mathrm{AU}$, as well as compares the absolute value of the highlatitude solar magnetic field component along the line of sight with the value total for both the hemispheres. Note that the last value has no meaning of flux but is a good indicator of the poloidal branch of solar activity. We can see that the magnetic flux through the photosphere behaves similarly to the toroidal branch (a maximum flux occurs during the period of maximum sunspot area), and the contribution of the dipole fields to this flux is small. Conversely, the magnetic flux through the source surface (or open SMF flux) is significantly shifted in time, its maximum is observed after the maximum sunspot area, and the contribution of the dipole fields to it is major (especially during periods of moderate and low sunspot activity).

The open flux is seen to correlate well with the HMF strength, but in the first years of the HMF dipole phase both the characteristics correlate better with the decaying SMF flux through the photosphere (i.e. with sunspot activity) than with increasing activity of the poloidal branch. In the second half of the HMF dipole phase, the situation changes. In this paper, to evaluate the effect of the poloidal branch of solar activity on the HMF flux, we use the idea that characteristics of this branch of solar activity during the epoch of solar minimum determine the next maximum of this cycle. In fact, this idea is the basis of a number of methods (beginning from [Ohl, 1966]) for forecasting maximum characteristics of sunspot cycle [Hathaway, 2015].

Consider first the correlation between the minimum value $B_{r}^{\min }$ and $S_{\mathrm{SS}}^{\max }$. To the left in Figure 8 is the regression of these characteristics. It is evident that the correlation is very high. Calculate now the coefficient of correlation between $\left|B_{r}\right|$ at $t$ and $S_{\mathrm{SS}}^{\max }$ and examine its time dependence. To the right in Figure 8 is the behavior of this coefficient as a function of time shift relative to the time of minimum of $\left|B_{r}\right|$. We can see that during the period $t-t\left(B_{r}^{\min }\right) \in[-2.5,1.5]$ the correlation coefficient is very high $(\rho>0.95)$, but before this period it quite dramatically decreases. We take this fact as an indication that in the period of high correlation between $\left|B_{r}\right|$ and $S_{\mathrm{SS}}^{\max } \mathrm{HMF}$ observed near Earth is determined mainly by the poloidal branch of solar activity. Note that this period is close to that when, according to the model [Bilenko, 2018], the contribution of poloidal SMFs in HMF observed near Earth exceeds the contribution of the toroidal branch SMF.

\section{DISCUSSION AND CONCLUSIONS}

Let us discuss first the apparent contradiction between the active role of HCS in forming SW velocity field (Subsection 4.3) and in identifying SW as a leading dynamic factor in the heliosphere (Section 2). The organizing role of HCS develops in the basement of the heliosphere, 


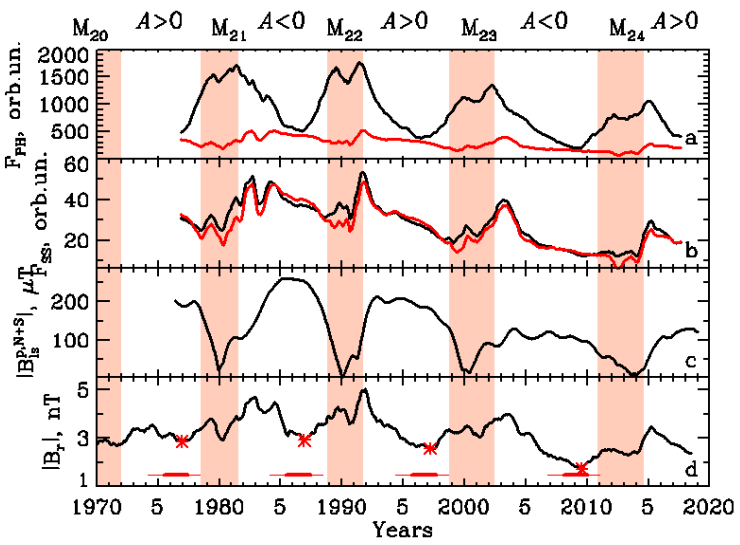

Figure 7. Magnetic flux through different layers of the solar atmosphere and poloidal solar field in 1970-2018. Vertical shaded stripes and text over the panels mean the same as in Figure 1. All the characteristics are 1-year smoothed. Solar magnetic fluxes are calculated using the radial version of the WSO model [http://wso.stanford.edu] without monopole term and are given in arbitrary units. Magnetic flux through the solar photosphere (black line) and contribution of dipole magnetic fields to this characteristic (gray line) $(a)$; the same as in panel $a$ but for a source surface $(b)$; absolute value of the highlatitude solar magnetic field component along the line of sight total for two hemispheres [http://wso.stanford.edu] (c); absolute value of the HMF radial component as derived from measurements at Earth's orbit [ftp://omniweb.gsfc.nasa.gov/pub/ data/omni/low_res_omni] $(d)$. In the bottom panel, asterisks mark minimum values of $\left|B_{r}\right|$, and horizontal lines show the periods (see Figure 8 ) when we associate this value with the poloidal branch of solar activity
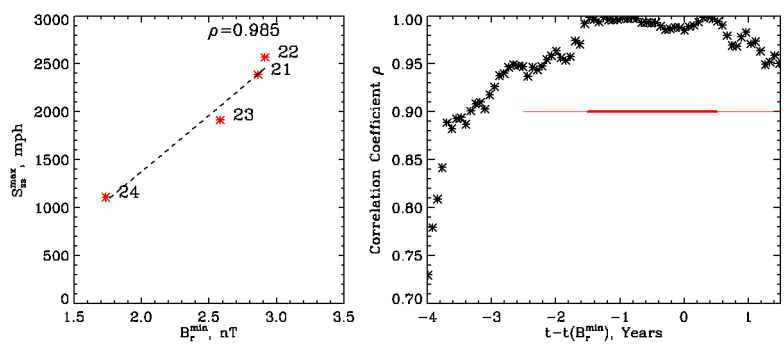

Figure 8. Correlation between the absolute value of nearEarth HMF radial component $\left|B_{r}\right|$ during low sunspot activity and the maximum value of this activity $S_{\mathrm{ss}}^{\max }$ (average for two Gnevyshev peaks, see Section 1 and Figure 1) in the next cycle for cycles 21-24. Correlation between minimum values $B_{r}$ and $S_{\mathrm{SS}}^{\max }$ (left); correlation coefficient between $B_{r}(t)$ and $S_{\mathrm{SS}}^{\max }$ as a function of time shift relative to the time of minimum of $B_{r}$ (right). The dashed line shows a period when $\rho>0.95$

where SMF is responsible for the separation of field lines into closed upon the photosphere and open to the heliosphere, along which SW, accelerated also in the basement, flows. In particular, under SMF neutral lines on the source surface there is a belt of closed field lines (helmet formations), and this determines the low SW velocity near HCS. In addition, for the lines open to the heliosphere SMF generates the geometry of flux tubes (the cross-section area as a function of distance along the tube), being responsible for the SW velocity increase with distance from HCS [Wang, Sheeley, 1990].
Indeed, the relative role of each of the branches of solar activity in heliospheric processes, particularly in GCR modulation, changes with solar cycle phase. During sunspot maximum, the toroidal branch is main for the heliosphere, the more so as in this period phenomena of the poloidal branch are very weak. In the early HMF dipole phase when the phenomena of the toroidal branch become weaker, whereas those of the poloidal branch become stronger, the effect of sunspot activity still prevails, at least, in the formation of HMF strength and spectrum of its irregularities. The role of this branch in forming the velocity field and global HCS form is not yet entirely clear. In most of the second half of the HMF dipole phase until the intensity of phenomena is maximum in the poloidal branch and minimum in the toroidal branch, the poloidal branch plays, in our opinion, the main role in the heliosphere (including the GCR modulation in it). Finally, at the end of the HMF dipole phase when the poloidal branch weakens and the toroidal one increases, the effect of both the branches on heliospheric processes again becomes comparable.

It is sometimes surprising why the conclusion about the importance of not only the toroidal branch but also of the poloidal one in heliospheric processes, including GCR modulation, seems so significant for us. In the end, whether it is really important for description of GCR behavior which solar activity features determine a certain phenomenon in the heliosphere (for example, generation of the global HCS or extremely inhomogeneous heliolatitudinal distribution of SW velocity) or not, if its characteristics and mechanisms of action on GCR are known. For us it is primarily a question of relating physics of different heliospheric layers, each with its own characteristics. Indeed, the heliosphere is one of the solar layers specially related to lower layers, and it may also involve processes of HMF transformation under the action of the field of velocities, this time SW velocities. In fact, these are HMFs in the Parker models which correspond to the development of the longitudinal, i.e. toroidal, component from the radial, i.e. poloidal, one under the action of non-rotating SW flowing radially and rotating HMF source surface. The turn of SW in the heliosheath to the heliospheric tail naturally leads to a change in all the three HMF components in this region.

Finally, we formulate conclusions of this paper.

- A significant role in forming and developing the heliospheric characteristics important for GCR propagation in it is played both by the toroidal branch of solar activity (active regions, sunspots and associated phenomena) and by the poloidal branch (coronal holes, high-latitude magnetic fields, zonal unipolar magnetic field regions), the role of each of the branches changing with solar cycle phase. The poloidal branch of solar activity determines the generation of the global HCS and its related distributions of SW velocity and HMF radial component, the formation of the region of interaction between multispeed SW streams. During low sunspot activity, the regular HMF strength is likely to depend on magnetic fields of the poloidal branch too.

- The more significant role of the poloidal branch in the heliosphere than in near-Sun layers of the solar 
atmosphere is due to the processes occurring in the layer located between them, where the main dynamic factor is SMF. Hence, during penetration of solar magnetic fields into the heliosphere larger-scale fields gain advantage and special SW and HMF characteristics are formed.

- As a result of the above heliospheric processes the main observed long-term variation of GCR intensity (11-year cycle) as well as the 22-year cyclicity are caused by both the branches of solar activity, the role of each of the branches changing with solar cycle phase. To determine the specific mechanism of this action requires a detailed simulation of GCR propagation in the heliosphere and additional calculations.

I am grateful to all teams of researchers presenting their findings on the Internet. This work was supported in part by RFBR grants No. 17-02-00584a, 18-02-00582a, 19-52-60003 SA-t.

\section{REFERENCES}

Ahluwalia H.S. Eleven year variation of cosmic ray intensity and solar polar field reversals. Proc. 16th ICRC. 1979, vol. 12, p. 182.

Altschuler M.D., Newkirk Jr. G. Magnetic fields and the structure of the solar corona. I. Methods of calculating coronal fields. Solar Phys. 1969, vol. 9, pp. 131-149. DOI: 10.1007/ BF00145734.

Arge C.N., Pizzo V.J. Improvement in the prediction of solar wind conditions using near-real time solar magnetic field updates. J. Geophys. Res. 2000, vol. 105, pp. 10465-10480. DOI: 10.1029/1999JA000262.

Balogh A., Jokipii J.R. The Heliospheric Magnetic Field and Its Extension to the Inner Heliosheath. Space Sci. Rev. 2009, vol. 143, pp. 85-110. DOI: 10.1007/s11214-008-9485-2.

Balogh A., Erdös G. The heliospheric magnetic field. Space Sci. Rev. 2013, vol. 176, iss. 1-4, pp. 177-215. DOI 10.1007/s11214-011-9835-3.

Balogh A., Hudson H., Petrovay K., von Steiger R. (eds.). Introduction to the solar activity cycle: overview of causes and consequences. Space Sci. Rev. 2014, vol. 186, iss. 1-4, pp. 115. DOI: $10.1007 / \mathrm{s} 11214-014-0125-8$.

Baranov V., Malama Y. Model of the solar wind interaction with the local interstellar medium: Numerical solution of selfconsistent problem. J. Geophys. Res. 1993, vol. 98, pp. 515715163.

Bazilevskaya G.A., Krainev M.B., Makhmutov V.S., Fluckiger E.O., Sladkova A.I., Storini M. Structure of the maximum phase of the solar cycles 21 and 22. Solar Phys. 2000, vol. 197, no. 1, pp. 157-174. DOI: 10.1023/A:1026515520311.

Bilenko I.A. Determination of the coronal and interplanetary magnetic field strength and radial profiles from largescale photospheric magnetic fields. Solar Phys. 2018, vol. 293 , iss. 7, article id. 106, 24 p. DOI: 10.1007/s11207-018-1324-31.

Bilenko I.A., ŁTavastsherna K.S. Coronal hole and solar global magnetic field evolution in 1976-2012. Solar Phys. 2016, vol. 291, pp. 2329-2352. DOI: 10.1007/s11207-016-0966-2.

Bruzek A., Durrant C.J. (Eds). Illustrated glossary for solar and solar-terrestrial physics. Dordrecht, D. Reidel Publ. Co. Astrophysics and Space Science Library. 1977, vol. 69, 224 p. DOI: 10.1007/978-94-010-1245-4.

Charbonneau P. Dynamo models of the solar cycle. Living Rev. Solar Phys. 2010, vol. 7, p. 3.

Dorman L.I. Variatsii kosmicheskikh luchei [Variations of Cosmic Rays]. Gostekhizdat Publ., 1957. 492 p. (In Russian).

Forbush S.E. Variation with period of two solar cycles in the cosmic-ray diurnal anisotropy and the superposed varia- tions correlated with magnetic activity. J. Geophys. Res. 1969, vol. 74, p. 3451.

Gerasimova S.K., Gololobov P.Yu., Grigoryev V.G., Krivoshapkin P.A., Krymsky G.F., Starodubtsev S.A. Heliospheric modulation of cosmic rays: model and observation, Solar-Terrestrial Phys. 2017, vol. 3, iss. 1, pp. 78-102. DOI: 10.12737/article_58f970f2455545.93154609.

Gnevyshev M.N. On the 11-years cycle of solar activity. Solar Phys. 1967, vol. 1, p. 107.

Hathaway D.H. The solar cycle. Living Rev. Solar Phys. 2015, vol. 12, p. 4. DOI: 10.1007/lrsp-2015-4.

Hoeksema J.T. Structure and evolution of the large scale solar and heliospheric magnetic fields. Ph.D. Thesis, Stanford University, USA, 1984, 222 p.

Hundhausen A.J. Coronal expansion and solar wind. Springer-Verlag Berlin-Heidelberg-New York, 1972, 238 p.

Jokipii J.R., Levy E.H., Hubbard W.B. Effects of particle drift on cosmic-ray transport. I. General properties, application to solar modulation. Astrophys. J. 1977, vol. 213, pp. 861-868. DOI: $10.1086 / 155218$.

Kalinin M.S., Krainev M.B. The formation of the sunspot and magnetic cycles in the GCR intensity in the heliosphere. J. Phys.: Conf. Ser. 2013, vol. 409, iss. 1, article id. 012156. DOI: 10.1088/1742-6596/409/1/012156.

Krainev M.B. Heliospheric long-term variations of galactic cosmic ray intensity. Baikal Young Scientists' International School on Fundamental Physics. XII Young Scientists' Conference "Interaction of Fields and Radiation with Matter". Irkutsk, 2013, pp. 17-20. (In Russian).

Krainev M.B. On the method of the GCR partial intensities related to the main physical processes of solar modulation. J. Phys.: Conf. Ser. 2015, vol. 632, no. 1, DOI: 10.1088/1742-6596/632/1/012061.

Krainev M.B. Causes of long-term variations in galactic cosmic ray intensity in the inner heliosphere // Bulletin of the Russian Academy of Sciences: Physics Allerton Press Inc., 2017, vol. 81, no. 2, pp. 166-169. DOI: 10.3103/s1062873817020241.

Krainev M.B., Kalinin M.S. The models of the infinitely thin global heliospheric current sheet. Proc. 12th Intern. Solar Wind Conf. Saint-Mal, 2009. AIP Conf. Proc., 2010, vol. 1216, pp. 371-374.

Krainev M.B., Kalinin M.S. On the description of the 11and 22-year cycles in the GCR intensity. J. Phys.: Conf. Ser. 2013a, vol. 409, iss. 1, article id. 012155. DOI: 10.1088/17426596/409/1/012155.

Krainev M.B., Kalinin M.S. On the GCR intensity and the inversion of the heliospheric magnetic field during the periods of the high solar activity. Proc. 33rd ICRC. Rio de Janeiro, Brasil, 2013b, ICRC2013-0317/1-4, arxiv:1411.7532 [astroph.sr], 2014.

Krainev M.B., Webber W.R. The solar cycle in the heliospheric parameters and galactic cosmic ray intensity. Multiwavelength investigations of solar activity. Proc. IAU Symp. No. 223. A.V. Stepanov, E.E. Benevolenskaya, A.G. Kosovichev, eds. Cambridge University Press, 2004, pp. 81-84. DOI: $10.1017 / \mathrm{s} 1743921304005150$.

Krainev M.B., Bazilevskaya G.A., Gerasimova S.K., Krivoshapkin P.A., Krymsky G.F., Starodubtsev S.A., Stozhkov YU.I., Svirzhevsky N.S. On the status of the sunspot and magnetic cycles in the galactic cosmic ray intensity. $J$. Phys.: Conf. Ser. 2013, vol. 409, iss. 1, DOI: 10.1088/17426596/409/1/012016

Krainev M., Kota J., Potgieter M.S. On the causes and mechanisms of the long-term variations in the GCR characteristics. Proc. 34th International Cosmic Ray Conference (ICRC2015). 2015. The Hague, the Netherlands. Online at http://pos.sissa.it/cgi-bin/reader/conf.cgi?confid=236, id.176. 
Krainev M.B., Bazilevskaya G.A., Kalinin M.S., Svirzhevsky N.S. On contribution of poloidal branch of solar activity to heliosphere and GCR modulation. IOP Conf. Ser.: J. Phys.: Conf. Ser. 2019, vol. 1181. 012010 IOP Publishing. DOI: $10.1088 / 1742-6596 / 1181 / 1 / 012010$.

Krymskiy G.F. Diffusion mechanism of diurnal cosmicray variations, Geomagnetism and Aeronomy, 1964, vol. 4, no. 6, pp. 763-769.

Lazar M. (ed.). Exploring the Solar Wind. Croatia, InTech Publ., 2012, 474 p.

Mackay D.H., Yeates A.R. The Sun's global photospheric and coronal magnetic fields: observations and models. Living Rev. Solar Phys. 2012, vol. 9, p. 6. DOI: 10.12942/lrsp-2012-6.

Ohl A.I. Forecast of sunspot maximum number of cycle 20. Solnechnye dannye [Solar Data], 1966, no. 12, p. 84. (In Russian).

Owens M.J., Forsyth R.J. The heliospheric magnetic field Living Rev. Solar Phys. 2013, vol. 10:5. DOI: 10.12942/lrsp2013-5.

Parker E.N. The passage of energetic charged particles through interplanetary space. Planet. Space Sci. 1965, vol. 13 , pp. 9-49. DOI: 10.1016/0032-0633(65)90131-5.

Parker E.N., Kennel C.F., Lanzerotti L.J. (eds.). Solar system plasma physics, V. 1 Solar and solar wind plasma physics, Amsterdam, North-Holland Publishing Co. 1979, 391 p.

Potgieter M.S. Solar Modulation of Cosmic Rays. Living Rev. Solar Phys. 2013, 10:3. DOI: 10.12942/lrsp-2013-3.

Richardson J.G. Solar wind stream interaction regions throughout the heliosphere. Living Rev. Solar Phys. 2018, vol 15:1. DOI: 10.1007/s41116-017-0011-z

Rosenberg R.L., Coleman Paul J., Jr. Heliographic latitude dependence of the dominant polarity of the interplanetary magnetic field. J. Geophys. Res. 1969, vol. 74, no. 24, p. 5611. DOI: 10.1029/JA074i024p05611.

Rossi B., Olbert S. Introduction to the Physics of Space. New York, McGraw Hill Publ., 1970.

Schatten K.H. Current sheet magnetic model for the sola corona. Cosmic Electrodynamics. 1971, vol. 2, pp. 232-245.

Schatten K.H., Wilcox J.M., Ness N.F. A model of interplanetary and coronal magnetic fields. Solar Phys. 1969, vol. 6, pp. 442-455.

Shulz M. Interplanetary sector structure and the heliomagnetic equator. Astrophys. Space Sci. 1973, vol. 24, p. 371 DOI: $10.1007 / \mathrm{BF} 02637162$
Smith E.J., Neugebauer M., Balough A., Bame S.J., Lepping R.P., Tsurutani B.T. Ulysses Observations of Latitude Gradients in the Heliospheric Magnetic Field: Radial Component and Variances. Adv. Space Res. 1995, vol. 72, iss. 9, pp. 165-170. DOI: 10.1016/0273-1177(95)00328-C

Stozhkov Yu.I., Svirzhevsky N.S., Bazilevskaya G.A., Svirzhevskaya A.K., Kvashnin A.N., Krainev M.B., Makhmutov V.S., Klochkova T.I. Fluxes of cosmic rays in the maximum of absorption curve in the atmosphere and at the atmosphere boundary (1957-2007) // Preprint LPI 2007, no. 14, $77 \mathrm{p}$.

Vainstein C.I., Zeldovich Ya.B., Ruzmaikin A.A. Turbulent Dynamo in Astrophysics, Moscow, Nauka, 1980, 354 p. (In Russian).

Wang Y.-M. Solar Cycle Variation of the Sun's Low-Order Magnetic Multipoles: Heliospheric Consequences. Space Sci Rev. 2014, vol. 186, pp. 387-407. DOI: 10.1007/s11214-014-0051-9.

Wang Y.-M., Sheeley N.R., Jr. Solar wind speed and coronal flux-tube expansion. Astrophys. J. 1990, vol. 355, p. 726. DOI: $10.1086 / 168805$.

Zhao X., Hoeksema J.T. A coronal magnetic field model with horizontal volume and sheet currents. Solar Phys. 1994, vol. 151, iss. 1, pp. 91-105. DOI: 10.1007/BF00654084.

URL: https://solarscience.msfc.nasa.gov (accessed February 6, 2019). ftp://ftp.swpc.noaa.gov/pub/forecasts/SRS/.

URL: http://wso.stanford.edu (accessed February 6, 2019).

URL: https://www.ngdc.noaa.gov/stp/space-weather/solardata/solar-imagery/composites/synoptic-maps/mc-intosh/ptmc _level3/ptmc_level3_gifs (accessed February 6, 2019).

URL: ftp://omniweb.gsfc.nasa.gov/pub/data/omni/low res_omni (accessed February 6, 2019).

URL: http://ufa.esac.esa.int/ufa/\#data (accessed February 6, 2019).

URL: https://sites.lebedev.ru/en/DNS_FIAN (accessed February 6, 2019).

How to cite this article

Krainev M.B. Manifestations of two branches of solar activity in the heliosphere and GCR intensity. Solar-Terrestrial Physics. 2019. Vol. 5. Iss. 4. P. 10-20. DOI: 10.12737/stp-54201902. 